\title{
Supernumerary Robotic Limbs for Human Augmentation in Overhead Assembly Tasks
}

\author{
Zack Bright, Harry H. Asada ${ }^{1}$
}

\begin{abstract}
Design and control of a novel extra robotic arm attached to the shoulder of a worker for performing tasks in the overhead area are presented. The wearable robot, a Supernumerary Robotic Limb (SRL), can lift an object and hold it while the wearer is securing the object using a tool with both hands. The worker does not have to take a laborious posture for a long time, reducing fatigue and injuries. Furthermore, a single worker can execute the task, which would otherwise require two workers. Two technical challenges and novel solutions are presented. One is to make the wearable robot simple and lightweight with use of a new type of Granular Jamming Gripper (GJG) that can grasp diverse objects from an arbitrary direction. This eliminates the need for orienting the gripper against the object with three-axis wrist joints, reducing the number of degrees of freedom (DOF) from 6 to 3. The other is an effective control algorithm that allows the wearer to move freely while the robot on the shoulder is holding an object. Unlike a robot sitting on a floor, the SRL worn by a human is disturbed by the movement of the wearer. An admittance-based control algorithm allows the robot to hold the object stably and securely despite the human movement and changes in posture. A 3 DOF prototype robot with a new GJG and an ergonomic body mounting gear is developed and tested. It is demonstrated that the robot can hold a large object securely in the overhead area despite the movement of the wearer while performing an assembly work.
\end{abstract}

Keywords- wearable robotics; extra robotic limbs; admittance control; Granular Jamming Gripper

\section{INTRODUCTION}

In the assembly of large commercial aircraft, one of the strenuous tasks is final assembly of fuselage internals, where workers must raise workpieces and components up to an overhead position and affix them to the ceiling or a wall. These tasks lead to some of the highest number of injuries from long term fatigue and wear, and thus to the most time away from work. Upper extremities are the most common injury site, and shoulders are $25 \%$ of those injuries. Overexertion and repetitive motion are especially strong contributors to this, with overexertion alone leading to 44 in 10,000 workers being injured annually [1].

The aircraft manufacturing industry is attempting to use exoskeletons, both passive and active, for assisting workers. Passive exoskeletons, including Lockheed-Martin's Fortis gear [3] and Robo-Mate's upper extremity support [4], can balance a heavy load with use of springs and ergonomically designed harness and linkages that distribute the load effectively to the wearer's body. Active upper-extremity exoskeletons have also been developed for lifting supports [5]. These include Cyberdyne's HAL[6], the especially ergonomic designs such as the UCLA 7-DOF arm [8] and the Schiel, van der Helm design [9], and many more that are outlined by Gopura et al. [7].

While these exoskeletons can augment the human strength in lifting and holding heavy items, the workers at aircraft fuselage assembly must do more than lifting and holding an object. Namely, most the tasks require the worker to affix workpieces and components while holding them at an overhead area. This often requires two workers working together in a confined space: one holding an object and the other securing it with a tool, e.g. Figure 12. shows assembly of an overhead compartment requiring two workers.

Supernumerary Robotic Limbs (SRL) aim to support workers with extra limbs. Unlike exoskeletons, SRLs can take an arbitrary posture independent of the human limbs, opening up new possibilities of assisting the human in close proximity. The SRL attached around the waist can hold an object while the worker is fixing it with a tool [11][12][13]. One attached to the chest can support the wearer in taking a crouching posture while working near the floor [14]. SRL fingers can assist hemiplegic patients in performing daily chores using an intuitive and implicit control method [15]. A pair of SRL arms on the shoulder has also been developed for assisting a worker in installing ceiling panels [16][17]. An extra robotic arm can even be used to play music and complete rhythmic tasks [18] These new types of wearable robots are a promising alternative, yet pose new challenges to overcome. SRLs must work effectively with the human in close proximity. Since the robot is attached to the human, the movement of the human may interfere with and disturb the robot [10]. The robot must be lightweight and compact as well as dexterous in holding various objects.

This paper presents a new design concept of SRL and its control algorithm. See Figure 12. Section II presents a key design concept that makes the wearable robot simple and lightweight, yet capable of grasping various objects in an arbitrary orientation without use of a servoed wrist. A new type of Granular Jamming Gripper (GJG) [20][21] is developed to eliminate the need for wrist DOF's yet allow for holding an arbitrary object on the ceiling. Section III addresses one of the key control challenges. Since the wearable robot is attached to the wearer's body, it is disturbed by human motion. To maintain a stationary position in space, e.g. holding an object on the ceiling, the robot must compensate for the human movement. Furthermore, the robot must allow the wearer to

\footnotetext{
*This work was supported by The Boeing Company.

${ }^{1}$ The authors are with the d'Arbeloff Laboratory for Information Systems and Technology in the Department of Mechanical Engineering, Massachusetts Institute of Technology, Cambridge, MA 02139, USA. Email: zbright@mit.edu; asada@mit.edu
} 


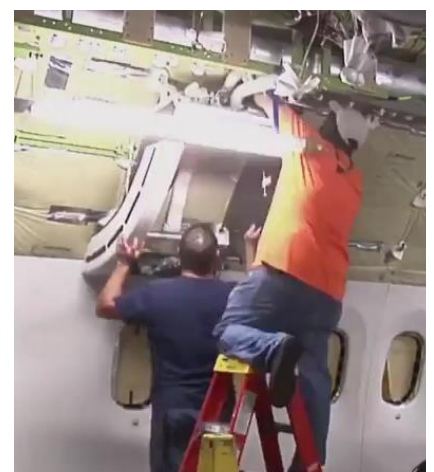

Figure 12. Workers Completing Overhead Tasks in Aircraft Assembly Snapshot of video of Boeing 787-9 Dreamliner Assembly [2]

move freely, for example, picking up a screwdriver and fixing the object with screws. Despite the human movement, the robot must hold the object stably. In the following section, an effective control algorithm based on admittance control will be presented to hold an object securely while allowing the wearer to change his/her posture without constraining the body. Section IV describes various practical issues that must be addressed to make the SRL design concept viable. These include ergonomic design of the human-robot attachment mechanism, safety measures, and actuators and power issues. Section $\mathrm{V}$ presents experimental verifications, followed by conclusion in Section VI.

\section{DESIGN CONCEPT}

\section{A. Functional Requirements}

The current work was motivated by the needs for assisting workers who have to perform assembly tasks in the overhead area for extended time periods. Raising the arms and keeping an overhead position are particularly fatiguing, since the muscle strength decreases as the arm is raised above the shoulder. A viable robotic assist system usable for these tasks must meet the following requirements:

- Access various sites of manufacturing environment together with an assembly worker; in aircraft manufacturing this requires a) moving across a cluttered area, b) climbing a steep staircase, and c) going through a narrow path and moving into a limited space;

- $\quad$ Reach a ceiling or an overhead area, and grasp various objects; in aircraft manufacturing, diverse objects ranging from stringers, frames, and various brackets to plates and boxes must be dealt with; and

- Securely hold an object by pressing it against the ceiling or a fixture in the overhead area.

\section{B. Shoulder-Mounted SRL}

Wearable robots, if designed properly, meet the functional requirements described above. A robot worn by a human worker can be transferred by the wearer, accessing various manufacturing sites together with the human. If the robot is separated from the worker, it faces hard mobility problems: it must avoid obstacles and go over numerous items scattered on the floor, climb a steep staircase of up to 45 degrees of grade, and move into a small space together with the worker.

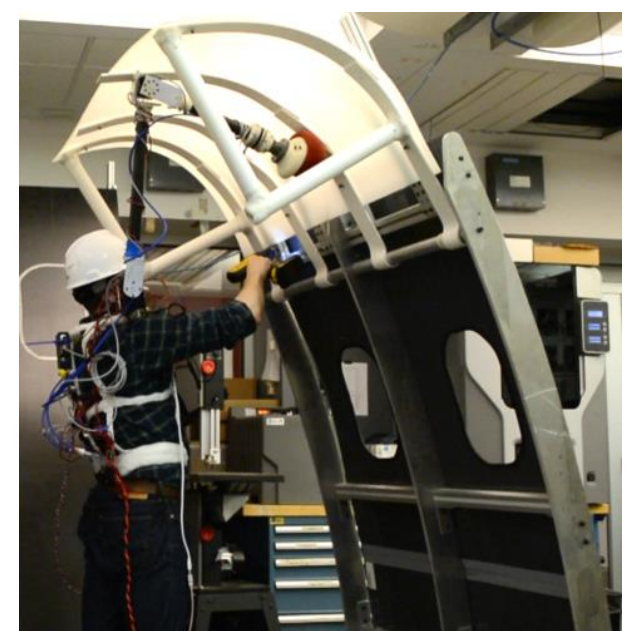

Figure 13. A person completing an overhead task with the SRL

The SRL approach can potentially eliminate these mobility and accessibility challenges, and provide a practical solution to using a collaborative robot in a cluttered manufacturing environment, which is difficult to access. As well, this means that the robot may leverage the flexibility, coordination, and planning of the wearer, without complex algorithms or interfacing.

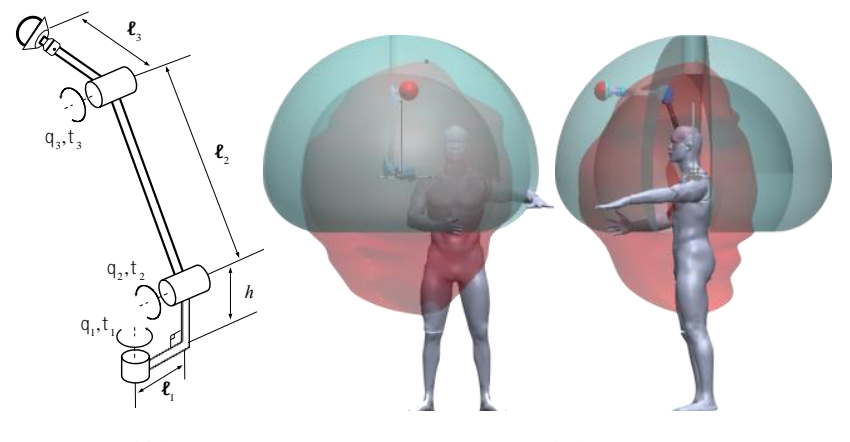

(A)

(B)

Figure 14. A) SRL Kinematic Structure and B) SRL Workspace (blue) with model of a person and the human arm workspace (red)

The SRL and specifically the shoulder-mounted SRL should meet other requirements, however. The robot must be lightweight and easy to carry around. Also, the robot must be ergonomically safe and comfortable to wear, as the wearer will bear both the weight and the forces applied by the SRL. With careful design of the interface, these loads can be distributed effectively to the entire body. Furthermore, the robot will be made lightweight by reducing the number of powered joints and using lightweight materials such as carbon fiber links and aluminum support brackets. The GJG described below is effective for reducing the number of joints as well as for grasping various objects.

\section{Use of a Granular Jamming Gripper}

To make the robot lightweight and simple to operate, we minimize the number of DOF. However, this poses the problem that the arm must be able to grasp objects of various dimensions and shapes and from different angles or postures of the robot. This has been accomplished with many versatile gripping strategies and types of grippers, ranging from under- 


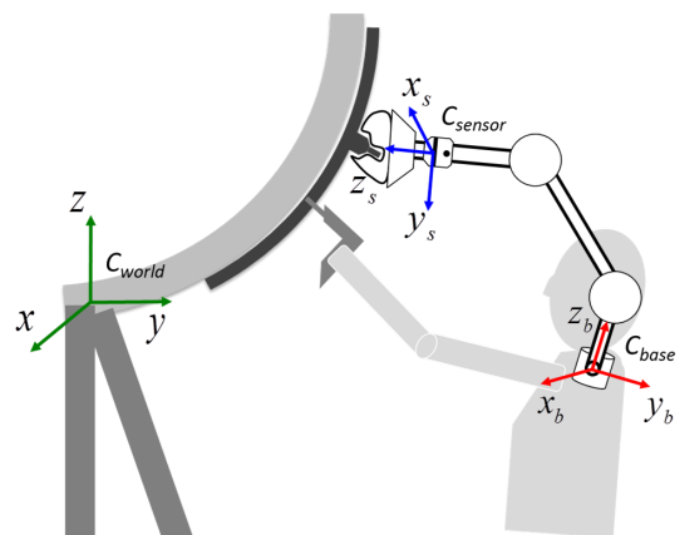

Figure 15. The Different Coordinate Systems in the Task Space

actuated grippers to modular to biomimetic grippers [20][21][22][23][24][25][26] however, in each of these cases the gripper is to be mounted on a high DOF arm that can position the gripper in $6 \mathrm{DOF}$ to allow for the correct grasping posture. We elicit the use of the universal GJG as proposed in [20][21]. Since the gripper not only adapts to any part geometry semi-passively, it can harden to shape without needing complex orientation. See Figure 5. Effectively, the end effector only needs to be pressed against the object and it can harden to grasp and support the object.

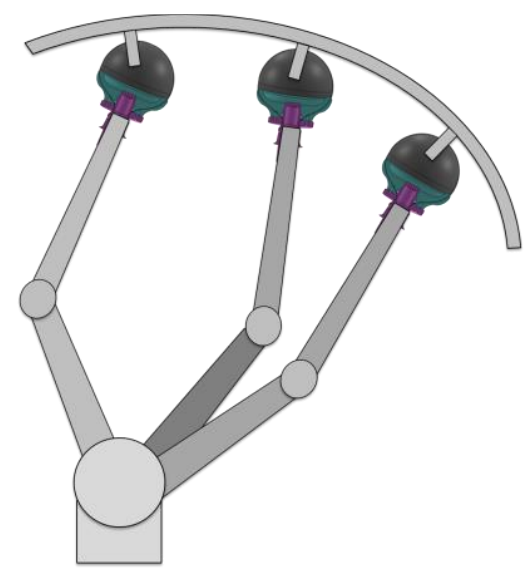

Figure 5. Granular Jamming Gripper can grasp objects from an arbitrary direction, eliminating the need for a powered wrist

For this specific application, we also note other advantages not otherwise explored in other literature involving GJGs:

- The jamming material is strongest under compression which is the typical use case in our supporting task

- By dynamically changing the internal pressure of the gripper, the jamming media can be treated as a stiff, highly damped fluid rather than a solid, fully-jammed media

- New design features of the gripper structure allow for increased force transmission in tension and in rotational shear

\section{Admittance Control for Human Movement COMPENSATION}

The SRL can be an effective solution, when multiple workers must work simultaneously in close proximity. In the aircraft fuselage assembly, as described previously, two workers must work side by side to install a large workpiece: one holding the workpiece and the other affixing it with a tool. The SRL can play the role of one worker holding the plate.

Despite the salient feature of the shoulder-mounted SRL, the control of the robot is inevitably more challenging. Since the SRL is mounted on the shoulder of a human, movements of the human continually disturb the robot. Unlike a robot sitting on a floor, or an assisting worker standing next to the main worker, the SRL is on a "moving base". As shown in Figure 4 the base coordinate frame $C_{\text {base }}-x_{b} y_{b} z_{b}$ is attached to the shoulder of the human where the SRL is secured. Viewed from the world coordinate frame $C_{\text {world }}-x y z$, the base frame moves as the human moves. The base movement acts as an exogenous disturbance to the control system of the SRL. The SRL control system must be able to compensate for the human movement disturbance.

Furthermore, in the context of the aircraft fuselage assembly, the worker will change the posture for picking up their tools, e.g. a screwdriver, and affix the plate being held by the SRL with fasteners. The SRL should not impede the human motion, but allow the human to move freely, while the plate must be held stably despite the human movements.

A naïve method for compensating for human movements is to measure the human position and orientation, and move the SRL in the opposite direction to the movement of the human. This method did not work for two reasons. One is that the human body motion is highly complex, and difficult to measure with a high spatiotemporal resolution. Second, since the object must be securely held with SRL, it must be pressed against a fixture or the ceiling. This requires controlling the force with which the SRL is holding and supporting the object at the tip. In general, force feedback loop needs a higher bandwidth, which is difficult to achieve with positional feedback signals.

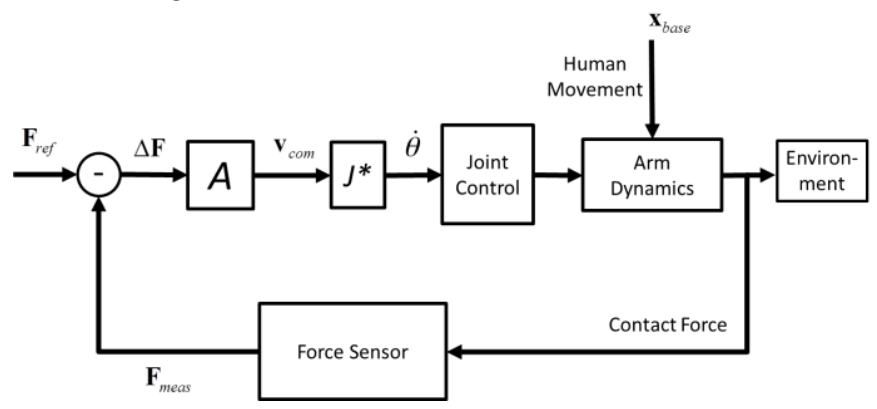

Figure 6. Force feedback with admittance control

Here we propose a force control method integrated with admittance control. Instead of measuring the human motion, we measure the force acting between the object being held and the wrist of the gripper, that is, the force that the SRL is applying to the object. To support the plate against gravity and assure that the plate is securely pressed against the ceiling or a fixture, the SRL must maintain a certain level of force or a 
reference force, $\mathbf{F}_{r e f}$, continuously. As shown in Figure 6. , a force feedback loop is formed around the endpoint force sensor. This force control system must not be disturbed by human movements, yet the SRL must accommodate the human desire to change the posture. To meet these requirements, we consider the following admittance control represented in the world coordinate system:

$$
\mathbf{v}_{\text {com }}=\mathbf{A} \cdot \Delta \mathbf{F}
$$

where $\mathbf{A} \in \mathfrak{R}^{3 \times 3}$ is an admittance matrix to be specified, $\mathbf{v}_{\text {com }} \in \mathfrak{R}^{3 \times 1}$ is a commanded velocity of the end-effector, and $\Delta \mathbf{F}$ is the difference between the reference and measured endpoint force:

$$
\Delta \mathbf{F}=\mathbf{F}_{\text {ref }}-\mathbf{F}_{\text {meas }}
$$

Suppose that the human attempts to move away from the gripping point of the object $\mathrm{P}_{\mathrm{G}}$. See Figure 13. Then the endpoint force of SRL tends to reduce, which is detected by the force sensor. This induces the velocity command $\mathbf{v}_{\text {com }}$ proportional to the reduction of the endpoint force, according to the admittance control law (1). The higher the admittance matrix value, the faster the SRL movement in pushing back the endpoint, so the endpoint force recovers. This is the simplest control algorithm that worked well, as demonstrated later in the experiment section. There are a few important points to remark, however.

Under-actuation: Recall that the shoulder-mounted SRL does not have a three-axis powered wrist. Thanks to the GJG, the SRL can grasp an object from an arbitrary direction, eliminating the need for a heavy powered wrist. This makes the SRL lighter and simpler, but it makes the system "underactuated". Fortunately, however, the GJG exhibits a significant rotational compliance with which it grasps an object. As the human attempts to move sideways, for example, the SRL responds to the change of the endpoint force and compensates for it by changing its configuration. This tends to change the orientation of the upper arm, resulting in a moment at the gripper. This resistive moment is small within $\pm 20^{\circ}$ or so, although it varies depending on the shape of the object and the suction pressure of the GJG.

Natural Haptic Feedback: It is interesting to note that the force applied by the SRL to the object is sensed by the human through the arm links and base of the SRL. Since the SRL is mounted on the shoulder of the human, all the reaction forces act on the human. This forms a "natural" haptic feedback from the contact point of the SRL to the human shoulder. Without using any active means, the human can sense and monitor the reaction force at the endpoint. In case the reaction force drops due to the human movement, it gives a warning to the human that the object being pressed against the ceiling is about being detached. Although difficult to quantify the human sensation, it has been observed through experiment, that the human can check whether the object is properly held and accommodate his/her motion to maintain the contact. We note that the admittance control itself intends to maintain a fixed force, and thus, the wearer should have a sense that constant force is applied.
Low-Level Control: Based on the admittance control law and the measurement of contact force $\mathbf{F}_{\text {meas }}$, the desired endpoint velocity $\mathbf{v}_{\text {com }}$ is determined in the world coordinate system. The SRL control system must be able to execute this velocity command faithfully. This is a standard low-level robot control problem, which has been addressed in the literature [19] However, a few critical implementation issues are worth mentioning. First, the velocity command $\mathbf{v}_{\text {com }}$ needs to be resolved to joint velocities with the Jacobian inverse. Near singularity, however, this causes an unwanted behavior. To alleviate this, the singularity-robust, inverse kinematics formulation is effective [22]. Second, a force feedback loop, in general, requires a higher sampling rate. In our case, the system tends to be unstable for an aggressively large admittance, which requires a large velocity for a small force discrepancy $\Delta \mathbf{F}$. Given a limited sampling rate of the force sensor, this may cause instability, as discussed further in the implementation and experiment sections.

Postural Compensation: As addressed earlier, the coordinate systems move with the human motion. To properly reference the force command, and maintain the force in the correct, global coordinates, we must compensate by tracking the human orientation. A 9DOF IMU is mounted to the base of the SRL and uses integrated sensor fusion to calculate the attitude of the robot base. From this measurement, we can transpose the forces measured in $\mathrm{C}_{\text {sensor }}$ into $\mathrm{C}_{\text {world }}$ and then compare to the reference force to get a desired velocity in $\mathrm{C}_{\text {world }}$, which is remapped back into $C_{\text {base }}$ to command the joint velocities in coordinate frame of the SRL.
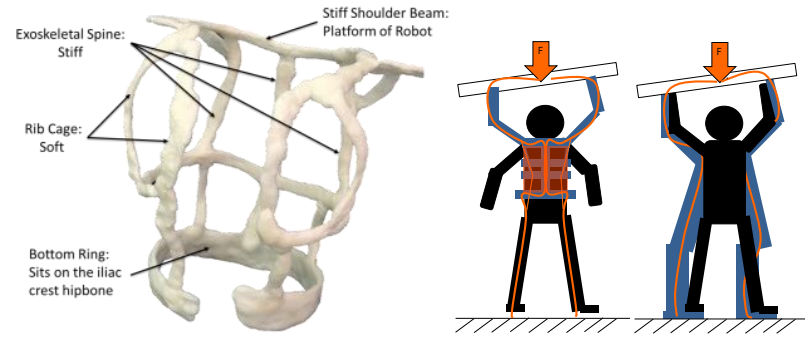

Figure 7. A) Ergonomic body attachment prototype and B) The structural loop of the new ergonomic attachment vs. a standard exoskeleton or person

\section{IMPLEMENTATION}

\section{A. Ergonomic Design of Body Attachment of SRL}

One of the critical design considerations for wearable robotics and more specifically in worn robotic limbs is the way load is transferred into the human wearer and how the robotic limbs are attached to the body. Ergonomic factors must be considered in transferring the load through the body without causing pain or discomfort. As well, we have brought into consideration the concept of haptic feedback that can be effectively proprioceptive for the wearer.

Both goals can be accomplished with a design that integrates stiff and compliant components. Figure 7. shows a prototype of body attachment structure made of thermoplastic materials and rods. A stiff platform across the shoulder that supports the robotic limb base is connected to an exoskeletal "spine" that is made to be very stiff in axial load, bending, and torsion. The spine is connected to a hip structure that can then 
transfer loads to the wearer. In addition to this simple, stiff base structure, there is another structure of less stiff components. This structure was inspired by the human ribcage, where the loads are by majority borne by the spine, but forces can be distributed across the torso to increase stiffness and reduce load on individual points along the spine. From this concept, we develop the structure shown in Figure 7.; the thin members act as the sternum and ribs of the design. These pieces of the structure are oversized in comparison to the wearer. The internal stresses here make the structure stiffer, and the compliance of the thermoplastic make this suit versatile to many body shapes and morphologies.

A key concept that makes this structure novel is the way the loads are distributed across the torso. We design the thin "rib" members such that they buckle under small deflections, pushing into the torso with even small strain at the robot base. This amplifies the strain, but transmits little load to the torso, allowing the wearer to have sensitivity to the forces applied by the robotic limbs while having little pressure or load borne on soft tissues, avoiding discomfort. For the purposes of making the suit iteratively and with tunable properties, we elect to use lightweight thermoform plastic that can be hand-molded, known as InstaMorph.

\section{B. Kinematic Design and Safety}

Thanks to the GJG, the number of degrees of freedom required for the arm reduced to 3 . This not only makes the robot design lightweight and compact, but can make it simpler to assure safety than for a full 6-DOF robot. The work space can be determined in a straightforward manner, and potential interferences with the human body, in particular, with the face can be evaluated without difficulty. Figure 14. shows the kinematic structure of the 3-DOF arm. The horizontal offset $\ell_{1}$ and the vertical offset $h$ at the first joint is for avoiding interferences with the face/head.

Figure 14. also shows the workspace of the prototype robot. The link lengths $\left(l_{1}=0.105, l_{2}=0.53, l_{1}=0.51, h=0.09\right)$ were optimized to be slightly longer than standard human reach and keep the workspace volume large yet distant from the worker's head and arms. Joint stops are used to prevent the workspace from extending into the human body. The key joint stop is that of the base joint, joint 1. As shown in Figure 14. , the head is kept outside of the volume of robotic workspace and the arm's end effector cannot reach to a point above the head of the wearer.

\section{Granular Jamming Gripper Prototype}

Figure 8. shows a prototype of GJG for the shoulder-mounted SRL. The gripper is made entirely of $3 \mathrm{~d}$ printed components from a traditional Fused Deposition Modeling (FDM) printer. Only the "head" of the gripper needs to be airtight; we achieve sufficiently airtight parts using post-processing of the FDM part. We employed acetone/solvent dipping as well as brushon epoxy sealing to get an airtight seal in the head.

The "cup" is comprised of two mirrored parts that compress the latex balloon onto the head to seal the system more tightly and to allow for the load borne by the gripper to transmit into the links of the robotic arm. Note that the geometry of the cup has a lip to capture the balloon structure as well as a smooth, splined surface that dogs with the jamming media when hardened. This shape is contrasted with the traditional form where the "cup" of the gripper is smooth and torque transmission relies on the material shear at the balloon-media interface as well as the adhesive seal between the 3d-printed "cup" and the outer surface of the latex balloon membrane.

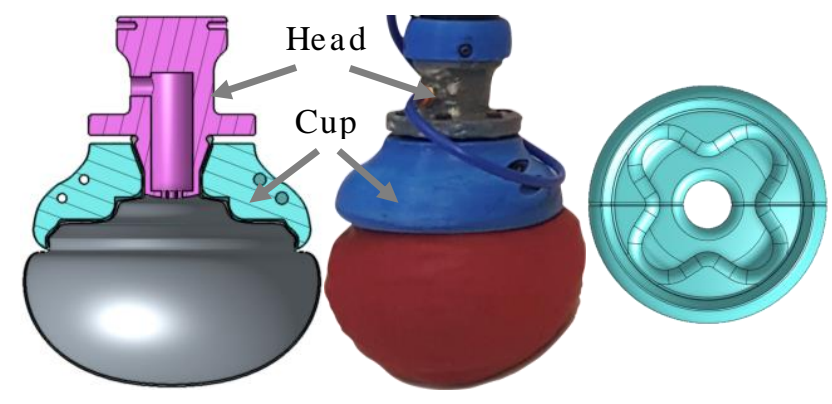

Figure 8. Granular Jamming Gripper Prototype and Cross-Section Model with bottom view of dogging pattern of cup

The granular jamming media used is a mix of hard Styrofoam pellets and ground coffee. This gives the high performance of coffee as a granular media, but is $10-20 \%$ the weight of the same volume of only coffee. Using a small $12 \mathrm{~V}$ DC diaphragm pump, we pull the needed 15 " $\mathrm{Hg}$ of vacuum for jamming, and up to 20 " $\mathrm{Hg}$. The pump also allows for positive pressure or inflation, using a valve system that connects the pump and GJG.

\section{Robotic Arm Prototype}

The current robot implementation is actuated by 3 Dynamixel Pro motors. These motors are controlled via serial communication by a USB2Dynamixel Dongle and a series of 4-pin RS485 cables. The structure is comprised of carbon fiber tube links, with 3D-printed couplings that join to aluminum motor mounting brackets. This robot arm is mounted to the ergonomic body attachment via another aluminum mounting bracket which is embedded into the thermoplastic exoskeleton. Our control system allows us to conservatively send motor commands and read joint position at $250 \mathrm{~Hz}$, read from the force sensor at $1000 \mathrm{~Hz}$, and read from the IMU at $100 \mathrm{~Hz}$. As the Dynamixels are capable of up to $70 \mathrm{~N} \cdot \mathrm{m}$ of torque at peak (rated $25 \mathrm{Nm}$ cont.), the SRL can hold roughly $80 \mathrm{~N}$ at full extension, but it the general workspace, if not constrained by software limits, it can apply $250-400 \mathrm{~N}$, this figure is of course dependent on configuration. The total mass of the arm prototype is $3.8 \mathrm{~kg}$, with the ergonomic attachment, battery, pump and other electronics on the full system is closer to 5.5 $\mathrm{kg}$, however, that weight is mostly borne by the hip, leaving this mass fairly non-apparent to the wearer.

\section{Testing AND Results}

Video of the tasks being completed can be found at: https://youtu.be/WWJXtVTjvcA

\section{A. Testing/Experimental Procedure}

There are many tasks for which the SRL is useful. Figure 12. shows the Overhead Assembly task, and Figure 16. shows the other tasks used to evaluate the SRL, the Crouching Support task, the Simple Force Holding task, and the Mock Tool Support task. These tasks were selected due to their commonplace presence in manufacturing settings. 
To clarify the specifics of each task and others done:

Overhead Assembly - The wearer sets up the manufacturing task with the SRL and uses the force control of the robot arm to support the overhead compartment while they affix the compartment with fasteners. During this task, the person must move around and back and forth, as well as change orientation, pick up or move tools, etc. The SRL must be able to maintain contact with and hold up the Overhead Compartment as the wearer bolts it in place. We simplify this task by starting from a configuration where the compartment has already been lifted by the worker, and can use some locating features to partially support the workpiece, as shown in Figure 12.

Crouching Support task - The worker is kneeling on the ground for some manufacturing task, e.g. cable routing, floor panel assembly, inspection, picking parts etc. The concept is that rather than the human supporting themselves with their hands, which would limit their dexterity and productivity, the SRL can hold the person up and allow both hands free to work. The wearer will shift their weight around and move up and down, side to side, and introduce oscillations to see if the SRL can stably support them. Success is qualified by the SRL supporting the worker without impeding work with instability.

Simple Force Holding task - The SRL gripper directly interfaces with a freestanding, sturdy structure. The force of the robot is directly loaded to the stiff structure. The wearer then moves around, back and forth and does so with varied movements, introducing oscillations to prompt frequency response, or rapidly moving away from the structure to see if the SRL will continue to correctly apply force. This task is meant for general data collection.

Mock Tool Support task - This task as mentioned before is meant to mimic the use of isoelastic arms in manufacturing tasks, such as the Fortis, or Robo-Mate. For our example, the robot supports a free mass of $2.27 \mathrm{~kg}$. The wearer will make the gripper hold the mass, then the wearer allows only the weight of the mass to apply force to the robot arm; the set point force is the weight of the mass, directed in the axis of gravity in the world coordinates, allowing for the weight to be supported and compensated for by the SRL. If the wearer inputs any additional force, the admittance controller acts to move the object in the direction of the disturbance force applied, making the "tool" weightless from the perspective of the wearer in the same vein as an isoelastic arm with the weight borne in the legs of the wearer.

An additional experiment was conducted to test the accuracy of the SRL's sensors and control to compensate and track the posture of the wearer. In this task, properties of the Singularity Robust Inverse Jacobian[22]were used to make the robot arm always point along the $\mathrm{X}$-axis. As the human wearer turns in Yaw as part of the trial, the robot must turn the base shoulder joint, $\theta_{1}$, to compensate. This gives us a direct measure of accuracy of the tracking in the control loop and the response time.

For all these procedures, we employ the same control procedure, intended to be used flexibly:
The wearer activates the SRL; The wearer moves the SRL into a starting position and presses or applies a setpoint force e.g. resting the free weight on the SRL while it is held up; Hold this position and force to allow the SRL to calibrate; Upon confirmation of calibration, release the SRL and begin the task; If ever there is safety risk, the wearer can trigger the E-Stop to force a shutdown.

\section{B. Human Haptic Feedback and Proprioception}

Very simple blind tests were performed to qualitatively explore the efficacy of the haptic feedback performance of the SRL. After some short time of practice and learning is undergone, the wearer can successfully identify the direction of force and torque on the end effector and can blindly touch to the end effector while blindfolded.

\section{Force Control Tracking and Holding}

During the various experiments and trials, the force applied to the structure, tool, workpiece etc. was maintained consistently, and the gripper remained in contact, applying force and never dropping or releasing the engagement as long as the admittance was tuned to the optimal value for the task. In Figure 9. note the highlighted regions which show that the deviation during most of the operation is roughly $\pm 2 \mathrm{~N}$. The large increases and decreases in force are from large changes in movement of the system, as the admittance controller commands velocity based off the force deviations.

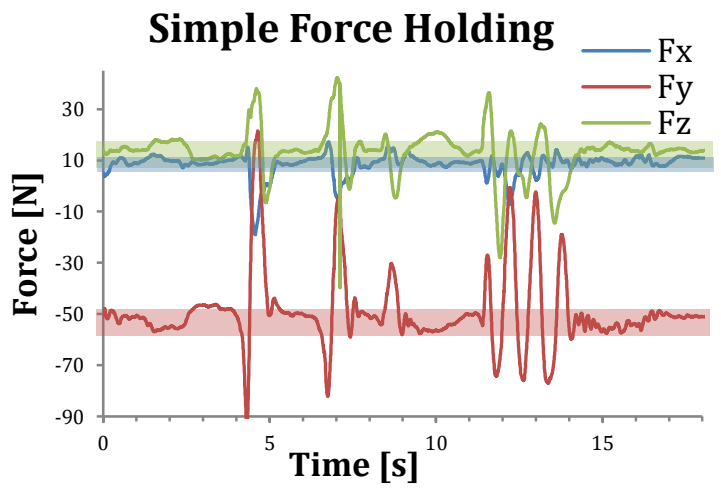

Figure 9. Force Measured During a Simple Force Holding Task (Admittance $=0.04 \mathrm{~m} / \mathrm{s} / \mathrm{N}$ ) In highlight: small deviations of only a few newtons unless the system is doing fast velocity compensation

The force data in the Overhead Compartment task shown in Figure 10. contains a similar trend of small deviations except in the case of large velocity disturbances. During the completion of the task, the robot arm stays engaged with the overhead compartment the entirety of the task. As well, no failures or releases were caused by large or fast movements by the wearer. The worker wearing the robot arm is not impeded by the position or motion of the robot arm either, and free motion is possible, as the worker could complete the entire manufacturing task easily.

Figure 14. shows the forces measured in the world coordinate frame during parts of each task as well; the dynamics of the control loop remain consistent throughout these trials, as the robot maintains good holding and applies 
consistent force without impeding human motion in all the tasks.

\section{E. Admittance Tuning}

By tuning the admittance of the control loop, one can tune the system for specific tasks that have different requirements. In systems where we would want the robot to be very damped and stiff, the system prevents the wearer from deviating from the correct position quickly. This is effective in some situations, but in general cases like ours, we prefer to allow free movement for the wearer.

As well, admittance controls are unstable if tuned too aggressively and can cause oscillations in the system by hitting self-resonance when under-damped. Therefore, we experimentally tune the admittance value in a series of force holding tasks to find a reasonable value for the admittance that gives good response time, close to what could be considered critical damping or slightly overdamped.

To test this, we repeat the Simple Force Holding task with various values of admittance. For our testing case the admittance matrix, $\boldsymbol{A}$, is a scalar matrix equal to $\boldsymbol{A I}$, where $A$ is the tuned scalar value of admittance in $\frac{m / s}{N}$. The wearer is instructed to rhythmically move back and forth to force the system with an input disturbance of some frequency to see if this causes instability or if the damping is too large at that point such that it restricts the movement of the wearer.

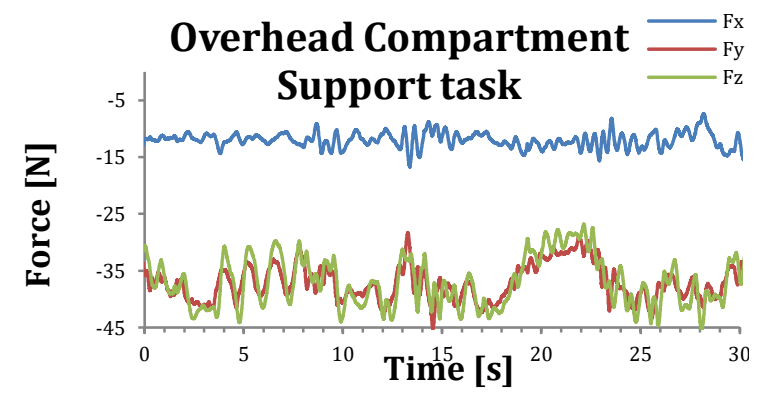

Figure 10. Force Measured During The Overhead Compartment Task (Admittance $=0.04 \mathrm{~m} / \mathrm{s} / \mathrm{N})$

Figure 11. shows the force output deviation from the set point force for various admittances, ranging from $0.10 \frac{\mathrm{m} / \mathrm{s}}{\mathrm{N}}$ to $0.013 \frac{\mathrm{m} / \mathrm{s}}{\mathrm{N}}$. For values of $A>0.05 \frac{\mathrm{m} / \mathrm{s}}{\mathrm{N}}$ the system is underdamped, and while the $0.1 \frac{\mathrm{m} / \mathrm{s}}{\mathrm{N}}$ controller has fast response with less force deviation, it begins to oscillate wildly when the wearer moves back and forth, this is from the inherent instability of the admittance controller, the admittance loop resonates with its output, causing greater measured force disturbance that it tries to compensate. For values of admittance below the optimally performing $0.04 \frac{\mathrm{m} / \mathrm{s}}{\mathrm{N}}$, the robot arm was overdamped. This was especially clear at $0.013 \frac{\mathrm{m} / \mathrm{s}}{\mathrm{N}}$ where the wearer was required to input an excessive amount of force to move the SRL. This is marked in the data by an increase in the amplitude and offset of the force deviation required to move the SRL, look to Figure 11. where the force deviations in $\mathrm{A}=0.02$ and $\mathrm{A}=0.013$ are larger in magnitude than those of the ultimately selected value of $0.04 \frac{\mathrm{m} / \mathrm{s}}{\mathrm{N}}$.

Around the optimal point of $0.04 \frac{\mathrm{m} / \mathrm{s}}{\mathrm{N}}$, we also test values of 0.033 and 0.05: in Figure 11. the amplitude of force deviation is again greater for the lower value of admittance, and, as well for some points the slightly increased admittance of $0.05 \frac{\mathrm{m} / \mathrm{s}}{\mathrm{N}}$ became unstable as shown in the highlighted region. We note then that we have found a well-balanced value of admittance at $0.04 \frac{\mathrm{m} / \mathrm{s}}{\mathrm{s}}$ as it is the highest admittance value tested that is not prone to instability, approximating our goal of near critical damping.
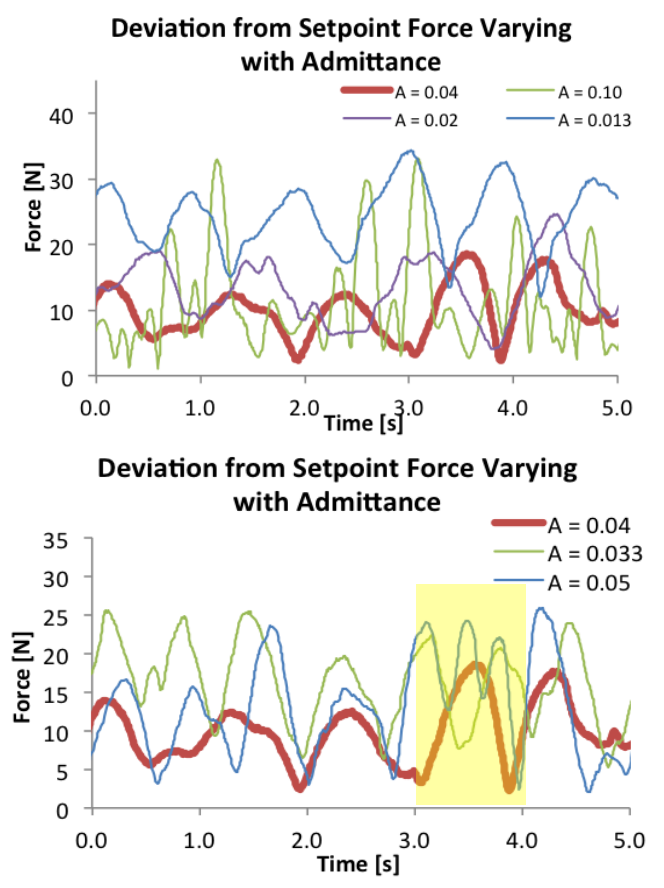

Figure 11. Forces Measured for Various Admittance Values, for the Tuning of the Admittance Parameter

\section{F. Postural Compensation and Tracking of the World Coordinates}

One of the major features of the control algorithm is to allow for the worker to move freely, and have the robot track

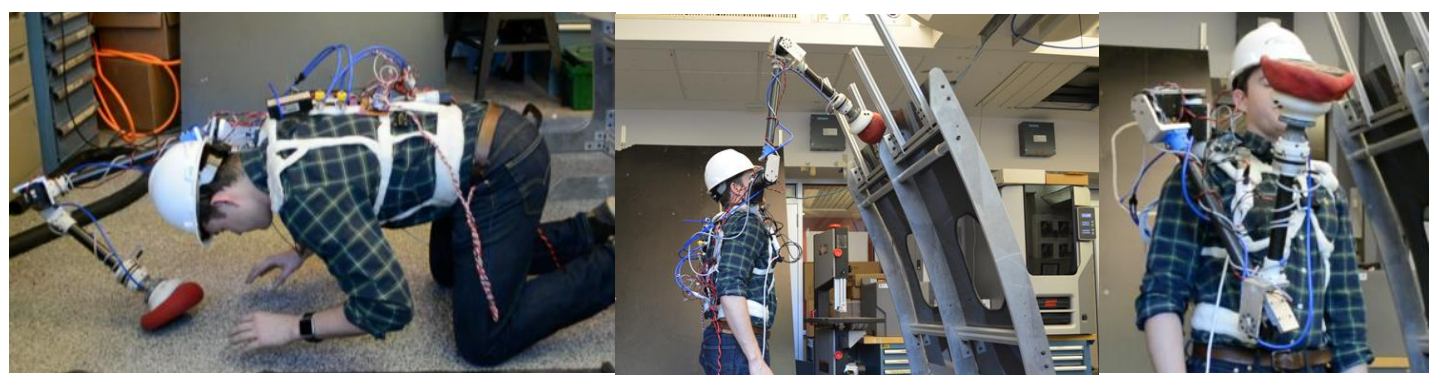

Figure 16. The Various Tasks: A) Crouching, Supported by SRL instead of own hands, B) Simple Force Holding, Pushing with Force Against Structure only, C) Supporting a Mass at the Gripper for Assisted Tool Holding or Manipulation 


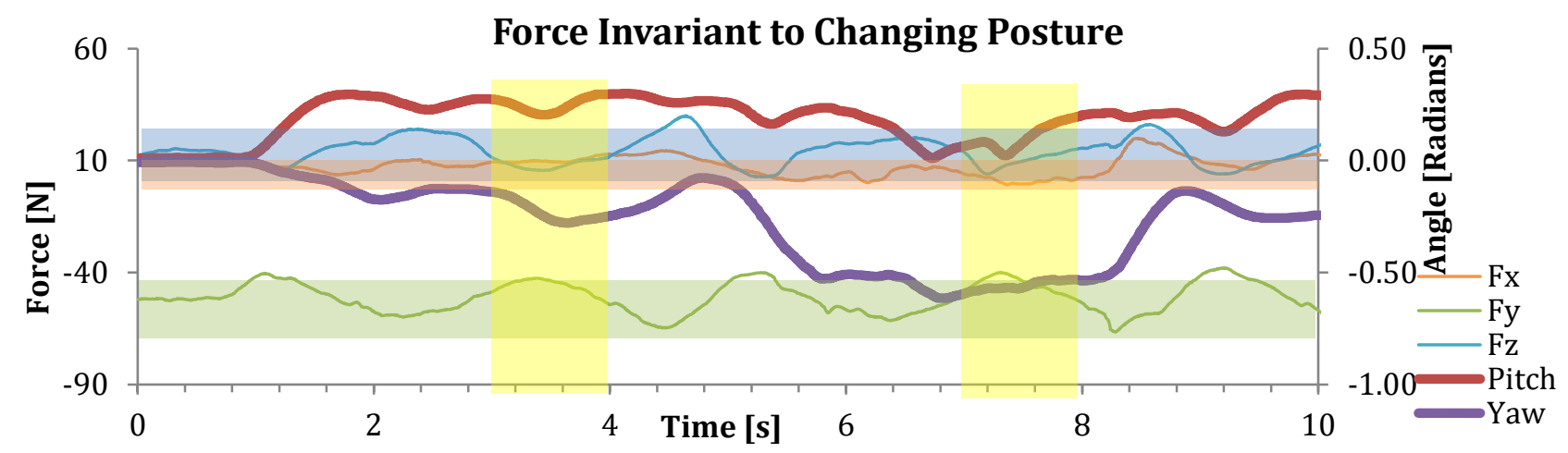

Figure 13. Force and IMU Data During a Force Holding Task

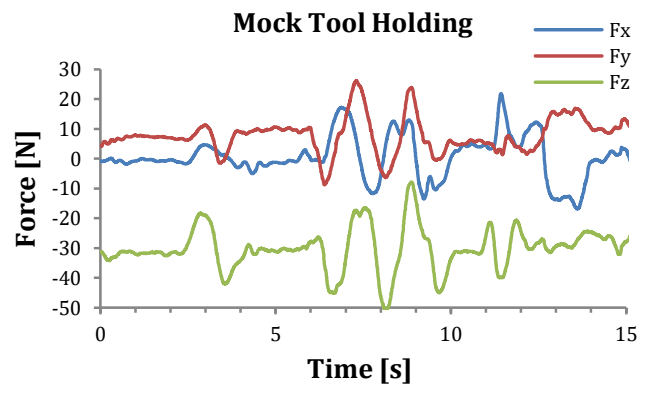

Figure 14. Force Measured During a Tool Supporting Trial $($ Admittance $=0.04 \mathrm{~m} / \mathrm{s} / \mathrm{N})$
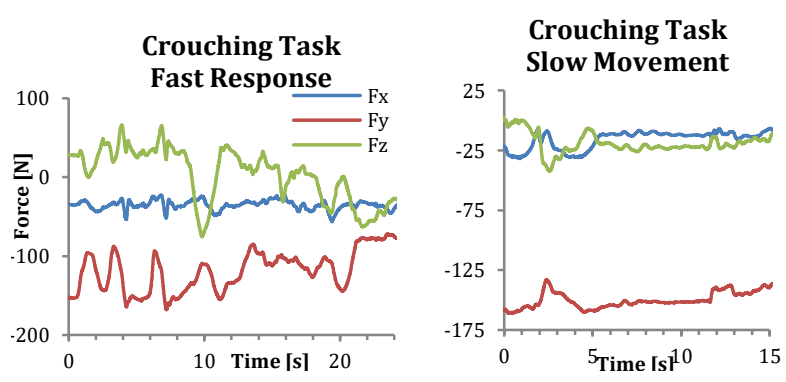

Figure 15. Force Measured During a Worker Prone Supporting Trial $($ Admittance $=0.04 \mathrm{~m} / \mathrm{s} / \mathrm{N})$

this motion to compensate and control the force applied in the direction of the world coordinate system specifically. The 9DOF IMU used allows the SRL control loop to track the roll, pitch, and yaw of the base of the robot and thereby the human worker. Figure 13. shows that in a force holding task, the orientation (being the pitch and yaw about the fixed axes) of the worker changes, yet the force in the world coordinates remains consistent.

Figure 16. shows data from a trial where the robot was attempting to stay in alignment with the $\mathrm{x}$-axis. As the wearer yaws or rotates about the $\mathrm{Z}$-axis, the shoulder joint, $\theta_{1}$ rotates to compensate. This is a strong indicator of good tracking as yaw is typically the hardest orientation to track with an IMU, as it is perpendicular to gravity, and so only the compass andthe accelerometer can track the yaw. This data shows little time delay and the offset is only a result of the mounting of the motor, being present since the start of the data collection. Our results also suggest that the control algorithm is effective at keeping the force compensation pointed in the world coordinate system. In Figure 13. the Pitch and Yaw vary over time, but when the forces are measured in the world coordinate frame, they only change to account for the velocity changes. Note the rapid change in yaw between 5 and 6 seconds. Here, no drastic change in the forces is observed, demonstrating effective compensation for postural changes when coupled with the data from Figure 16. The colored bands in Figure 13. show the range of values and highlight that the amplitude or offset of the oscillations do not change with changing Pitch or Yaw. Compare the regions of 3-4s vs $7-8 \mathrm{~s}$ where the difference in Yaw is $\sim 0.30 \mathrm{rad}$ or almost $20^{\circ}$.

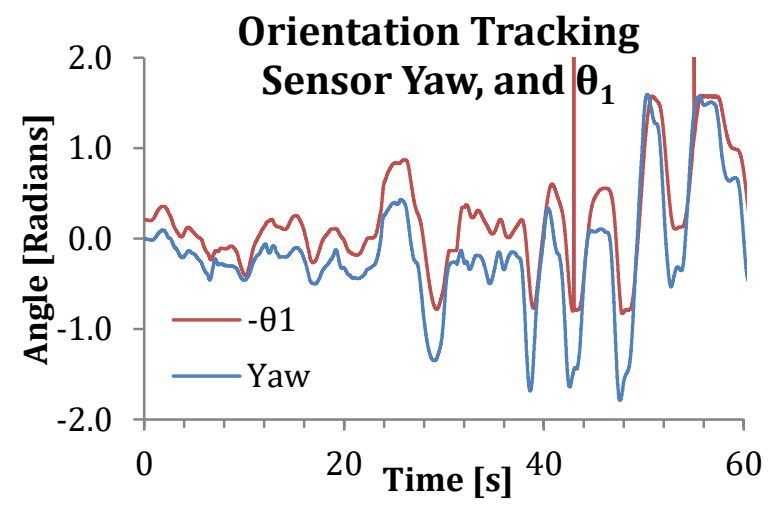

Figure 16. Orientation and Position Data During Vector Following Trial; the two angles are complimentary: as $\theta 1$ rotates the shoulder to compensate for the change in Yaw. Shown here is the Yaw angle and negative $\theta 1$

\section{CONCLUSION}

An improved design of Supernumerary Robotic Limbs is presented in addition to the design of a novel ergonomic attachment that is used to interface the SRL with the wearer. We also present a novel use case and some design changes to the universal Granular Jamming Gripper. A closed loop implementation of force control allows the wearer to move and perform dynamic tasks while the SRL holds workpieces overhead as well as accomplish many other tasks. The control scheme presented yields a natural and intuitive interface for SRL interaction that is also effective and versatile. Other future work to be explored may include tighter integration of this force control scheme with direct/inferred control, and increased control complexity. As well, we believe that future work can allow for direct control using similarly intuitive feedback to give more natural interactions that make the SRLs more akin to an extension of the human body as opposed to a worn robotic arm, aiming for intuitive human augmentation. 


\section{REFERENCES}

[1] Bureau of Labor Statistics, "2015 Nonfatal Occupational Injuries and Illnesses: Cases with days away from work", Bureau of Labor Statistics, 2016.

[2] British Airways. "British Airways - Building the 787-9 Dreamliner." Online video clip. YouTube. YouTube, Sep 30, 2015. Web. 15 Nov, 2015.

[3] Lockheed Martin. FORTIS EXOSKELETON - RELIEF FOR THE DAILY GRIND. N.p.: Lockheed Martin, 2016. Lockheed Martin, 2016. Web. 15 Jan. 2017.

[4] Altenburger, Ruprecht, Daniel Scherly, and Konrad S. Stadler. "Design of a Passive, Iso-elastic Upper Limb Exoskeleton for Gravity Compensation." ROBOMECH Journal 3.1 (2016).

[5] L. Martin, "Relief for the Daily Grind: Industrial Exoskeletons at Work", 2014.

[6] "CYBERDYNE." The World's First Cyborg-type Robot "HAL®" - CYBERDYNE. CYBERDYNE Inc., 2017. Web. 15 Jan. 2017.

[7] R. A. R. C. Gopura and K. Kiguchi, "Mechanical designs of active upper-limb exoskeleton robots: Stateof-the-art and design difficulties," 2009 IEEE International Conference on Rehabilitation Robotics, Kyoto International Conference Center, 2009, pp. 178187.

[8] J. C. Perry and J. Rosen, "Design of a 7 Degree-ofFreedom Upper-Limb Powered Exoskeleton," The First IEEE/RAS-EMBS International Conference on Biomedical Robotics and Biomechatronics, 2006. BioRob 2006., Pisa, 2006, pp. 805-810.

[9] A. Schiele and F. C. T. van der Helm, "Kinematic Design to Improve Ergonomics in Human Machine Interaction," in IEEE Transactions on Neural Systems and Rehabilitation Engineering, vol. 14, no. 4, pp. 456469, Dec. 2006.

[10] F. Parietti and H. Asada, "Dynamic analysis and state estimation for wearable robotic limbs subject to humaninduced disturbances," in Proc. IEEE Int. Conf. Robot. Autom., 2013, pp. 3880-3887.

[11] F. Parietti and H. Asada, "Supernumerary robotic limbs for aircraft fuse- lage assembly: Body stabilization and guidance by bracing," in Proc. IEEE Int. Conf. Robot. Autom., 2014, pp. 1176-1183.

[12] F. Parietti and H.Asada, "Supernumerary Robotic Limbs for Human Body Support", IEEE Transactions on Robotics, Vol.32, No.2, pp301-311, 2016.

[13] F. Parietti, K. Chan, and H. Asada, "Bracing the human body with supernumerary robotic limbs for physical assistance and load reduction," in Proc. IEEE Int. Conf. Robot. Autom., 2014, pp. 141-148.

[14] D. Kurek and H. Asada, "The MantisBot: Design and Impedance Control of Supernumerary Robotic Limbs for Near-Ground Work", to appear in Proceedings of the 2017 IEEE International Conference on Robotics and Automation, 2017

[15] F. Wu and H. Asada, "Implicit and Intuitive Grasp Posture Control for Wearable Robotic Fingers: A Data-
Driven Method Using Partial Least Squares", IEEE

Transactions on Robotics, vol. 32, no. 1, pp. 176-186, 2016.

[16] B. L. Bonilla and H. H. Asada, "A robot on the shoulder: Coordinated human-wearable robot control using Coloured Petri Nets and Partial Least Squares predictions," International Conference on Robotics and

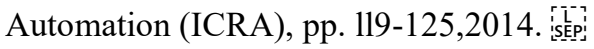

[17] Llorens-Bonilla, B., Parietti, F., and Asada, H. H., "Demonstration-based control of supernumerary robotic limbs," In Intelligent Robots and Systems (IROS), 2012 IEEE/RSJ International Conference on. IEEE, pp. 39363942, 2012.

[18] Georgia Tech. "Robot allows musicians to become three-armed drummers." Online video clip. YouTube. YouTube, Feb 17, 2016. Web. Mar 6, 2016.

[19] B. Siciliano and O. Khatib (ed.), "Handbook of Robotics", Part A Robotics Foundations, Springer, 2008.

[20] E. Brown, N. Rodenberg, J. Amend, A. Mozeika, E. Steltz, M. Zakin, H. Lipson, H. M. Jaegera, "Universal robotic gripper based on the jamming of granular material", PNAS, 107(44): pp. 18809-18814, 2010.

[21] J. Amend, E. Brown, N. Rodenberg, H. Jaeger and H. Lipson, "A Positive Pressure Universal Gripper Based on the Jamming of Granular Material", IEEE

Transactions on Robotics, vol. 28, no. 2, pp. 341-350, 2012.

[22] N. G. Cheng et al., "Design and Analysis of a Robust, Low-cost, Highly Articulated manipulator enabled by jamming of granular media," 2012 IEEE International Conference on Robotics and Automation, Saint Paul, MN, 2012, pp. 4328-4333.

[23] J. Kapadia and M. Yim, "Design and performance of nubbed fluidizing jamming grippers, " 2012 IEEE International Conference on Robotics and Automation, Saint Paul, MN, 2012, pp. 5301-5306.

[24] T. Nishida, D. Shigehisa, N. Kawashima and K. Tadakuma, "Development of universal jamming gripper with a force feedback mechanism," 2014 Joint 7th International Conference on Soft Computing and Intelligent Systems (SCIS) and 15th International Symposium on Advanced Intelligent Systems (ISIS), Kitakyushu, 2014, pp. 242-246.

[25] R. R. Ma, L. U. Odhner and A. M. Dollar, "A modular, open-source 3D printed underactuated hand," 2013 IEEE International Conference on Robotics and Automation, Karlsruhe, 2013, pp. 2737-2743.

[26] G. P. Jung, J. S. Koh and K. J. Cho, "Underactuated Adaptive Gripper Using Flexural Buckling," in IEEE Transactions on Robotics, vol. 29, no. 6, pp. 1396-1407, Dec. 2013. 
[27] Y. Nakamura and H. Hanafusa, "Inverse Kinematic Solutions With Singularity Robustness for Robot Manipulator Control", Journal of Dynamic Systems, Measurement, and Control, vol. 108, no. 3, p. 163, 1986. 